\title{
Effects of enriched environment and probiotics on the intestinal mucosal barrier and the brain-gut axis in rats with colorectal cancer
}

\section{Dun Liu}

Fujian Medical Universtiy

Wu Xian-Yi (D wuxianyi1973@163.com )

Huang Si-Ting

Fujian Medical University

\section{Research}

Keywords: Barrier, colorectal cancer, intestinal mucosa, enriched environment, probiotic

Posted Date: May 12th, 2020

DOI: https://doi.org/10.21203/rs.3.rs-26783/v1

License: (c) (i) This work is licensed under a Creative Commons Attribution 4.0 International License. Read Full License 


\section{Abstract \\ Background}

Enriched environment is a paradigm where animals are introduced to novel, complex, and stimulating surroundings that can protect the intestinal mucosal barrier and regulate the expression of brain-gut peptides. Probiotics can effectively protect the intestinal mucosal barrier and regulate brain-gut axis activity in colorectal cancer patients. This study assessed the effects of probiotics, enriched environment, and joint intervention on the intestinal mucosal barrier and brain-gut axis in rats with colorectal cancer.

\section{Methods}

We used a rat model of 1,2-dimethylhydrazine-induced colorectal cancer. Rats were housed in four different conditions for 2 weeks: enriched environment, probiotic,joint condition and normal condition. Each rat was weighed, and the intestinal mucosa and plasma levels of tumor TNF-a, IL-6, IL-10, ghrelin, CRF, occludin, BT, SIgA and the morphology of the intestinal mucosa were measured.

\section{Results}

enriched environment was beneficial regarding bacteria translation, plasma and intestinal mucosa levels of cytokines, plasma CRF levels, villi length and width of intestinal mucosa and hypothalamus ghrelin compared to probiotic $(P<0.05)$. There were no statistical differences between the enriched environment and the other groups regarding occludin, SIgA, muscle thickness or intestinal mucosa ghrelin $(P>0.05)$.

\section{Conclusions}

The effect of enriched environment was better than probiotic, especially in the intestinal mucosal immune and biological barrier in rats with colorectal cancer. However, the combination of the two was not as effective as enriched environment. In future studies, we can investigate the role of environment and probiotics in SIgA, intestinal mucosal mechanical barrier and body weight by extending the intervention time and enlarging the sample size.

\section{Backgroud}

The enriched environment $(\mathrm{EE})$ is a paradigm whereby animals are introduced to novel, complex, and stimulating surroundings that promote structural changes in the brain and enhance learning and memory performance in rodents ${ }^{[1]}$. In an EE, mice or rats are placed in larger cages containing multiple physical and social stimuli, which allow the animals to explore, exercise, and interact with each other ${ }^{[2]}$. Recent studies have investigated the role of EEs in cancer treatment and prognosis ${ }^{[2-5]}$. Several studies have 
indicated that EEs can regulate the intestinal microenvironment ${ }^{[6]}$ and enhance intestinal immunity ${ }^{[7-11]}$, thereby protecting the intestinal mucosal barrier. In addition, EEs can regulate the expression of brain-gut peptides ${ }^{[12-15]}$, thus regulating gastrointestinal function. Furthermore, some studies have revealed a positive correlation between colorectal cancer (CRC) and certain commensal bacteria ${ }^{[16,17]}$. Several reports have also shown that probiotics/prebiotics can alleviate and treat some of the clinical symptoms of $\mathrm{CRC}$ by protecting the intestinal mucosal barrier function; regulating intestinal secretion, intestinal microflora, intestinal epithelial cell reproduction, and intestinal immunity; and by inhibiting bacterial translocation (BT) and reducing toxic substances ${ }^{[18-21]}$. Moreover, some studies ${ }^{[22,23]}$ have shown that probiotics can also influence brain cognition, reverse disease-induced brain dysfunction, and modulate brain-gut axis activity. In summary, probiotics can effectively protect the intestinal mucosal barrier and regulate brain-gut axis activity in CRC patients, thereby inhibiting the occurrence and development of tumors, enhancing the therapeutic effect and promoting rehabilitation of patients. But the effect of EE on intestinal mucosal barrier in colorectal cancer is not clear. Therefore, in this study, CRC rats were treated with probiotics, $\mathrm{EE}$, and joint intervention of probiotics and $\mathrm{EE}$, and comparative analyses were carried out in order to understand the effects of environmental enrichment on the intestinal mucosal barrier in rats with $\mathrm{CRC}$, and to lay a foundation for studying the effects of rehabilitation, environmental care, and dietary care on the body.

\section{Material And Methods}

\section{Animals}

All procedures were approved by the Laboratory of Animal Welfare and Ethics Committee of the University (certificate number 2016-06) and were performed in accordance with the National Institute of Health guidelines for the treatment of animals. We obtained 48 male four-week-old Sprague-Dawley rats, weighing approximately 150-160 g each, from the Animal Experimental Center of Fujian Medical University. Rats were housed in ventilated cages each containing four to five rats with soft shavings and air-conditioning $\left(22 \pm 1{ }^{\circ} \mathrm{C}, 50-60 \%\right.$ humidity, $12 \mathrm{~h}$ light-dark cycles), and were fed ad libitum (20-25\% protein, $5-10 \%$ fat, $3-5 \%$ crude fiber). The food was prepared and mixed according to the guidelines set by the Association of Analytical Communities. Prior to experiment, rats were fed adaptively by the criteria described above for two weeks. Animals were then monitored for agility and missing teeth. Rats that showed neither occurrence were deemed fit to proceed with the investigation.

\section{Generation Of 1,2-dimethylhydrazine-induced Tumors}

From six weeks of age, DMH (Sigma Chemical Co., USA) was injected subcutaneously (concentration $2 \%$, $20 \mathrm{mg} / \mathrm{kg}, \mathrm{pH} 6.5$ ) into the rats once a week for 21 weeks. DMH is highly carcinogenic and has high organ specificity, which means it can specifically induce colorectal cancer. DMH has been used in many studies for modeling colorectal cancer in rats ${ }^{[24]}$. 


\section{Animal Housing Procedures}

At 21 weeks after injection with $\mathrm{DMH}$, all rats were examined by ultrasonography (Esaote, MylabClassC, Italy; probe frequency $18-22 \mathrm{MHz}$ ). Tumor formation occurred in all 48 rats; these were then divided into four groups of 12 rats each, by using stratified randomization grouping according to their weight. The main evidence is that previous studies have shown an elevated level of prostaglandin E2 in the colon mucosa of patients with colorectal polyps or colorectal cancer ${ }^{[25]}$. Prostaglandin E2 is related to the progression of malignant tumor and intestinal barrier function ${ }^{[26]}$. Body mass index (BMI) affects the level of prostaglandin E2. The higher the BMI, the higher the level of prostaglandin E2 ${ }^{[27]}$. In addition, body weight measurement is non-invasive, which will not cause adverse effects on rats. Therefore, in this study, weight can be used as a baseline measure of tumor progression and cancer bowel function.

For EE conditions, large cages $(109 \times 79 \times 41 \mathrm{~cm})$ of twelve rats were used. The set-up method of EE was taken from the relevant literature ${ }^{[28-31]}: \otimes$ The number of rats in an EE is typically $6-20$ per cage. $\otimes$ The spatial size of an EE has been in the range $30,000-50,000 \mathrm{~cm}^{3}$ per rat. $\otimes$ The number of stimulatory objects has not been specified in the reported studies; however, it can be surmised that the number of stimulatory objects was about 1-2 per rat. $\otimes$ The types of objects included huts made of wood, walking wheels made of plastic with a diameter of 21 centimeters, transparent labyrinths tunnels made of acrylic with a diameter of 13 centimeters and various wooden toys. All of these objects were harmless to rats. Items destroyed by the rats were replaced periodically. In addition, the positions of the stimulants, water, and food in the cages were changed twice weekly to ensure the freshness of the rats' environment. For the probiotics $(P)$ group, three standard cages $(54.5 \times 39.5 \times 20 \mathrm{~cm})$ were used with four rats each, and without any stimulating objects. Rats were given $2 \mathrm{ml}\left(1.0 \times 10^{9}\right.$ colony-forming units [CFU]) of probiotic powder orally for 14 days. This contained three components (Kangning, Shenzhen, China): Bifidobacterium lactis (strain number HN019), Bifidobacterium lactis (strain number Bi-07), and Lactobacillus rhamnosus (strain number HN001). The probiotic powder was dissolved in $2 \mathrm{ml}$ pure water at $37^{\circ} \mathrm{C}$. The administration method for the probiotics was taken from the relevant literature ${ }^{\text {[32] }}$. Studies have shown that in the intervention of rat probiotics, $1 * 10^{9} \mathrm{CFU} /$ day only for high concentration of probiotics, $1 * 10^{8} \mathrm{CFU} /$ day only for low concentration of probiotics ${ }^{[32]}$.

Studies have also shown that the use of high concentration probiotics is more conducive to the protection of intestinal mucosal barrier function ${ }^{[33]}$. For the probiotics and $E E(P E)$ group, twelve rats were reared in a large cage and given probiotics by oral administration, as described above. In the blank (B) group, rats were housed in three standard cages with four rats each, without any stimulating objects. After two weeks of experimentation, two rats had died from the PE group and P group, respectively. Large abdominal masses were found after the death of the rats. Therefore, it was presumed that the cause of death was the tumor burden.

\section{Western Blotting}


The brain-gut peptide ghrelin was detected by western blotting. Prior to obtaining tissues samples, all rats were anesthetized then sacrificed. Colon tissue (approximately $100 \mathrm{mg}$ ) was clipped at $2 \mathrm{~cm}$ from the end of the cecum and washed with saline. Tissues were then placed immediately in an Eppendorf tube and kept frozen at $-80^{\circ} \mathrm{C}$. About $100 \mathrm{mg}$ tissue was treated with $200 \mu$ Protein Seeker Mammalian Cell Lysis Solution (GenDEPOT). The mixture was ground with ice water, shaken at $1200 \mathrm{rpm}$ for $5 \mathrm{~s}$ and then centrifuged at $12,000-16,000 \mathrm{rpm}$ for $5 \mathrm{~min}$. Supernatants were collected and stored at $-80{ }^{\circ} \mathrm{C}$ prior to use. Extracted proteins were analyzed by sodium dodecyl sulfate polyacrylamide gel electrophoresis and then transferred onto a polyvinylidene fluoride membrane (Pall). Western blots were performed using a polyclonal anti-ghrelin primary antibody (1:250; Abcam, UK). Membranes were then incubated with horseradish peroxidase-conjugated secondary antibodies (1:8000, ZB-2301, Zhongshan Company, Beijing) for $1 \mathrm{~h}$. Immunoreacted proteins were detected using the ECL-Plus Western Blotting Detection System (Amersham Life Sciences, Braunschweig, Germany).

\section{Measurement of TNF-a, IL-6, IL-10, and corticotropin-releasing factor levels}

Rat intestines (approximately $400 \mathrm{mg}$ of tissue per sample) were clipped and washed with saline. Samples were cut into slices, homogenized using a Dounce homogenizer (WHEATON, USA), and centrifuged at $4{ }^{\circ} \mathrm{C}$ and $10,000 \mathrm{rpm}$ for $30 \mathrm{~min}$. Supernatants from each fraction were collected and stored at $-80^{\circ} \mathrm{C}$. Brain tissues were isolated in an ice bath, and the hypothalamus was separated and rapidly placed in an Eppendorf tube. Tissues were then frozen in liquid nitrogen for 5 min and stored at $-80^{\circ} \mathrm{C}$. For serum samples, blood was drawn from the inner canthus vein. Whole blood was incubated at $4{ }^{\circ} \mathrm{C}$ for $24 \mathrm{~h}$ and then centrifuged at $10,000 \mathrm{rpm}$ for $10 \mathrm{~min}$. The serum was isolated from whole blood using a liquid transfer gun and stored at $-80^{\circ} \mathrm{C}$. All samples were then thawed and the levels of TNF-a, IL6, IL-10, and CRF were determined by enzyme-linked immunosorbent assay (ELISA) according to the manufacturer's instructions.

\section{Detection Of Bacterial Translocation}

Mesenteric lymph nodes, livers, and spleens were collected under sterile conditions. To each specimen, $1 \mathrm{ml}$ cold saline was added. Samples were ground using a mortar and pestle, and $0.5 \mathrm{ml}$ of each sample was incubated with medium containing eosin methylene blue agar at $37^{\circ} \mathrm{C}$ for $48 \mathrm{~h}$. The rosin acid contained in the medium only inhibits the growth of Gram-positive bacteria but has no inhibitory effect on the growth of Gram-negative bacteria. Bacteria with lactose decomposition will form blue colonies on the medium. Escherichia coli is a Gram-negative bacterium with lactose decomposition, which forms blue colonies on this medium, with the size of a single colony being about $2 \mathrm{~mm}^{\text {[34] }}$. The number of bacterial colonies was counted and the number of CFU per gram of tissue (CFU/g) was calculated. In this study, bacteria were cultured as Enterobacteriaceae, and the number of blue colonies in culture plates was more than five colonies indicated a positive result ${ }^{[35]}$. 


\section{Intestinal Mucosa Morphology}

Rat intestines were isolated and fixed with $10 \%$ formaldehyde, dehydrated, and embedded in paraffin. Sections of $5 \mu \mathrm{m}$ thickness were cut, dewaxed with xylene, hydrated with an alcohol gradient, and stained with hematoxylin for $1 \mathrm{~min}$. Samples were washed with phosphate-buffered saline (PBS) and stained with eosin for $15 \mathrm{~s}$, then rapidly dehydrated with an alcohol gradient. Finally, sections were treated with xylene, mounted in neutral gum, and viewed with a light microscope. Hematoxylin and eosin (HE) staining results were evaluated using Image-Pro Plus 6.0 software, and groups were compared.

\section{Immunohistochemical Detection Of Occludin And Secretory Immunoglobulin A}

Rat intestines were fixed in $10 \%$ formalin for $24 \mathrm{~h}$ and embedded in paraffin. Sections of $5-\mu \mathrm{m}$ thickness were cut, mounted on slides, and incubated with anti-occludin (1:120, Thermo, USA) or anti-SIgA antibodies (1:100, Thermo) for $2 \mathrm{~h}$ at $37^{\circ} \mathrm{C}$. Slides were then washed three times with PBS and incubated with goat anti-rabbit secondary antibodies (Maixin Biological Technology Development Co, Ltd, Fuzhou, China) for $30 \mathrm{~min}$. Slides were washed three times with PBS and developed using diaminobenzidine color development solution (Fuzhou Maixin Biotechnology Development Co, Ltd) for $5 \mathrm{~min}$. Slides were then stained with hematoxylin for 1 min, washed with PBS, dehydrated with an alcohol gradient, treated with xylene, mounted with neutral gum, and viewed with a light microscope (Nikon SMZ645, Japan). Immunohistochemical staining results were evaluated using Image-Pro Plus 6.0 software, and betweengroup comparisons were conducted. The investigator who analyzed all immunohistochemically-stained slides was blinded to the group allocation of each sample. The expression level analysis of occludin and SIgA was carried out according to the relevant literature ${ }^{[36]}$.

\section{Weight}

There was no interaction between the intervention effect and the time effect $(F=0.75, P=0.611)$. There were no significant differences among different intervention groups $(F=0.292, P=0.839)$ or different time points $(F=1.908, P=0.155)$.

\section{Statistical analysis}

BT values measured in various organs are presented in the result as percentages. The other indices are presented as means \pm standard deviation. Differences between groups were assessed using two-factor analysis of variance (ANOVA) with a post-hoc Bonferroni pairwise comparison. Weight differences were assessed using two-way classification repeated ANOVA. For non-normal distribution of data, a KruskalWallis test with post-hoc Mann-Whitney U test for pairwise comparison was performed. For all statistical analyses, $\mathrm{P}<0.05$ was considered to be statistically significant. An adjusted significance level of $\mathrm{P}<0.01$ 
was used for post-hoc pairwise comparisons. All statistical analyses were performed using SPSS 24.0 statistics software (SPSS Inc, Chicago, IL, USA).

\section{Results}

\section{Microscopic morphology of intestinal mucosa Villus length}

The samples were collected from rat intestines and pathological methods were used for detection. There was a significant effect of the combined $E E$ and probiotics intervention on small intestinal villus length ( $F$ $=10.643, P=0.002)$. Small intestinal villus length showed a significant difference upon $E E$ intervention $(F$ $=26.600, P<0.001)$, but no significant difference upon probiotics intervention $(F=0.365, P=0.549)$. The villus length of group $B$ is $133.271 \pm 14.569 \mu \mathrm{m}$, that of group $P$ is $149.452 \pm 15.434 \mu \mathrm{m}$, that of group EP is $157.383 \pm 14.791 \mu \mathrm{m}$ and that of group EE is $168.507 \pm 11.826 \mu \mathrm{m}$. Further comparison analysis showed that there were statistically significant differences between group $B$ and the other groups. There were no statistical differences among the other groups(Figure 1).

\section{Villus Width}

The samples were collected from rat intestines and pathological methods were used for detection. There was no significant effect of the combined $E E$ and probiotics intervention on small intestinal villus width $(F=3.030, P=0.089)$. Small intestinal villus width showed a significant difference upon $E E$ intervention $(F=10.516, P=0.002)$, but no significant difference upon probiotics intervention $(F=0.194, P=0.662)$. The villus width of group $B$ is $131.173 \pm 11.263 \mu \mathrm{m}$, that of group $P$ is $143.057 \pm 12.093 \mu \mathrm{m}$, that of group $\mathrm{EP}$ is $151.241 \pm 22.061 \mu \mathrm{m}$ and that of group $\mathrm{EE}$ is $158.323 \pm 24.463 \mu \mathrm{m}$. Further comparison analysis showed that there were statistically significant differences between group $B$ and group $E E(P=0.006)$, but no statistical differences among the other groups (Figure 1).

\section{Muscle Layer Thickness}

The samples were collected from rat intestines and pathological methods were used for detection. There was no significant effect of the combined $E E$ and probiotics intervention on muscle layer thickness $(F=$ $0.168, P=0.684)$. Muscle layer thickness showed a significant difference upon $E E$ intervention $(F=7.931$, $P=0.007)$, but no significant difference upon probiotics intervention $(F=0.001, P=0.982)$. The muscle layer thickness of group $B$ is $157.068 \pm 20.836 \mu \mathrm{m}$, that of group $P$ is $159.104 \pm 18.245 \mu \mathrm{m}$, that of group EP is $170.414 \pm 15.579 \mu \mathrm{m}$ and that of group EE is $172.232 \pm 3.293 \mu \mathrm{m}$. Further comparison analysis showed that there were no statistical differences among the four groups $(F=2.738, P=0.055)$ (Figure 1$)$. 


\section{Immunohistochemical Detection Of Occludin}

The samples were collected from rat intestines and immunohistochemical methods were used for detection. There was no significant effect of the combined $\mathrm{EE}$ and probiotics intervention on occludin levels $(F=2.816, P=0.101)$. Occludin showed no significant difference upon $E E$ intervention $(F=0.244, P$ $=0.624)$ or probiotics intervention $(F=0.100, P=0.754)$ alone. The occludin of group $B$ is $1.42 \pm 1.505$, that of group $P$ is $2.18 \pm 1.25$, that of group EP is $1.73 \pm 1.191$ and that of group EE is $2.25 \pm 1.215$. Further comparison analysis showed that the differences in occludin levels among the four groups were not significant $(F=1.079, P=0.368)$.

\section{Plasma TNF-a, IL-6, And IL-10 Levels}

The samples were collected from serum. Elisa methods were used for detection.

There was a significant effect of the combined $\mathrm{EE}$ and probiotics intervention on the plasma levels of IL$10(F=555.804, P=0.000)$. Plasma levels of IL-10 showed no significant difference upon $E E$ intervention $(F=0.939, P=0.338)$, but there was a significant difference upon probiotics intervention $(F=9.532, P=$ 0.004). Further comparison analysis showed that there was no significant difference between the $P$ group and the EE group $(P=0.457)$. The differences among the other groups were statistically significant(Figure 2).

There was a significant effect of the combined EE and probiotics intervention on the plasma levels of IL- 6 $(F=33.325, P=0.000)$. The plasma levels of IL-6 showed a significant difference upon both $E E$ intervention $(F=397.285, P=0.000)$ and probiotics intervention $(F=280.181, P=0.000)$ alone. Further comparison analysis showed that the difference in the plasma IL- 6 levels between the PE group and the $B$ group was not statistically significant $(P=0.119)$, but that there were significant statistical differences among the other groups(Figure 2).

There was no significant effect of the combined $\mathrm{EE}$ and probiotics intervention on the plasma levels of TNF- $a(F=26.669, P=0.000)$. The plasma levels of TNF-a showed a significant difference upon EE intervention $(F=1601.716, P=0.000)$ and probiotics intervention $(F=288.331, P=0.000)$ alone. Further comparison analysis showed that the differences in the plasma TNF-a levels among the four groups were statistically significant (Table 1, Fig. 2).

\section{TNF-a, IL-6, And IL-10 Levels In The Intestinal Mucosa}

The samples were collected from rat intestines. Elisa methods were used for detection.

There was a significant effect of the combined $\mathrm{EE}$ and probiotics intervention on the intestinal mucosal levels of IL-10 ( $F=75.803, P=0.000)$. The intestinal mucosa levels of IL-10 showed a significant difference upon $E E$ intervention $(F=151.672, P=0.000)$ and probiotics intervention $(F=4.448, P=0.041)$ 
separately. Further comparison analysis showed that the difference in intestinal mucosal IL-10 levels between group $\mathrm{P}$ and group PE was not statistically significant. The differences between the other groups were significant(Figure 2).

There was a significant effect of the combined EE and probiotics intervention on the intestinal mucosal levels of IL-6 $(F=5.272, P=0.027)$. The intestinal mucosa levels of IL- 6 showed a significant difference upon $E E$ intervention $(F=1244.744,0.000)$ and probiotics intervention $(F=111.2865, P=0.000)$ separately. Further comparison analysis showed that the differences in the intestinal mucosal IL- 6 levels among the four groups were significant(Figure 2).

There was also a significant effect of the combined EE and probiotics intervention on the intestinal mucosal levels of TNF-a $(F=262.126, P=0.000)$. The intestinal mucosal levels of TNF-a showed a significant difference upon $E E$ intervention $(F=79.855, P=0.000)$, but no significant difference upon probiotics intervention $(F=0.101, P=0.752)$. Further comparison analysis showed that the differences in the intestinal mucosal TNF-a levels among the four groups were significant (Table 1, Fig. 2).

\section{Immunohistochemical Detection Of SIgA}

The samples were collected from rat intestines and Immunohistochemical methods were used for detection. There was no significant effect of the combined EE and probiotics intervention on SIgA levels $(F=0.810, P=0.373)$. SlgA showed no significant differences upon $E E$ intervention $(F=0.176, P=0.677)$ or probiotics intervention $(F=1.587, P=0.215)$ alone. Further comparison analysis showed that the differences in SlgA among the four groups were not significant (Table 2).

\section{BT Ratio In Four Groups}

The results indicated that BT occurred in 20 out of 36 tissues in the EE group, 25 out of 33 tissues in the PE group, 29 of 33 tissues in the $P$ group, and 27 of 36 tissues in the B group. According to double-factor ANOVA, there was no effect of the combined $E E$ and probiotics intervention $(F=0.245, P=0.621)$ in response to $B T$. There was a significant difference upon $E E$ intervention $(F=4.558, P=0.035)$ and probiotics intervention $(F=5.007, P=0.027)$ in response to $B T$. Further comparison analysis showed that the difference between group $P$ and group $E E$ was statistically significant $(P=0.013)$. There were no statistical significant differences among the other groups.

\section{Brain-gut Peptide Levels In Rats With CRC Plasma CRF levels in rats with CRC}

There was a significant effect of the combined $E E$ and probiotics intervention on the plasma levels of CRF $(F=28.516, P=0.000)$. Plasma levels of $C R F$ showed a significant difference upon $E E$ intervention ( $F$ 
$=51.801, P=0.000)$, but no significant difference upon probiotics intervention $(F=2.034, P=0.161)$.

Further comparison analysis showed that the difference in plasma CRF levels between group $\mathrm{P}$ and group PE was not significant. There were significant differences between the other groups (Table 3, Fig. 3).

\section{Ghrelin Secretion In The Hypothalamus}

There was no significant effect of the combined $\mathrm{EE}$ and probiotics intervention on ghrelin secretion in the hypothalamus $(F=1.089, P=0.303)$. Ghrelin secretion in the hypothalamus showed a significant difference upon $E E$ intervention $(F=12.361, P=0.001)$ or probiotics intervention $(F=6.090, P=0.018)$ alone. Further comparison analysis showed that there were significant differences between group $B$ and group EE, and between group $B$ and group PE. Differences between the other groups were not significant $(F=6.628, P=0.001)($ Table 4, Fig. 4).

Figure 4. Ghrelin secretion in the hypothalamus.

\section{Ghrelin Secretion In The Intestinal Mucosa}

There was no significant effect of the combined $\mathrm{EE}$ and probiotics intervention on ghrelin secretion in the intestinal mucosa $(F=1.828, P=0.184)$. Ghrelin secretion in the intestinal mucosa showed a significant difference upon $E E$ intervention $(F=1.142, P=0.291)$ or probiotics intervention $(F=2.578, P=0.116)$ alone. Further comparison analysis showed that there were no significant differences between the four groups $(F=1.893, P=0.145)$ (Table 4, Fig. 4).

\section{Discussion}

Many studies have shown that intestinal microorganisms can confer healthy benefits on their host. Probiotics are involved in regulating intestinal flora, immunity, and the mucosal barrier ${ }^{[37]}$. Moreover, some studies have suggested that an important consequence of a modified bacterial community could be a change in the expression of a range of different bacterial genes in the bowel contents, as well as in the intestinal mucosa of the host. Analogous observations with probiotics, the stimulation of cytokines, and modification of immune responses could be important in producing beneficial effects ${ }^{[38]}$. On the other hand, the EE, which promotes "eustress" or positive psycho-social stress ${ }^{[5]}$, not only influences brain structure and function ${ }^{[39]}$, but also significantly inhibits tumor growth in syngeneic melanoma, colon cancer ${ }^{[5]}$, and breast cancer models ${ }^{[4]}$. Moreover, some studies have indicated that physical exercise influences potential preventive pathways in the colon mucosa, reducing colon cancer risk [21]. Therefore, EE can protect the intestinal mucosal barrier of CRC patients, thereby inhibiting tumor growth to a certain extent. Therefore, the comparative study of probiotics and EE can be used to explore their protective effects on the intestinal mucosal barrier, and also to explore the effects of combined intervention on the intestinal mucosal barrier in colorectal carcinoma. 


\section{Intestinal Mucosal Mechanical Barrier}

Intestinal epithelial cells and tight junctions (TJs) between intestinal epithelial cells form the structural basis of the intestinal mucosal mechanical barrier. The TJ barrier function can also be affected by changes in the distribution of specific TJ proteins and/or their expression levels. The intestinal epithelial transmembrane binding protein occludin is one of the main closely connected proteins which is transmembrane protein engaged in zonulae occludens. It has been proven to affect the permeation of ions and soluble substances and is involved in regulating the migration of cell bypass immune cells [ ${ }^{40-}$ 42]. Therefore, the combination of occludin with the length, thickness, and muscular thickness of intestinal epithelial villi can affect the intestinal mucosal mechanical barrier to a certain extent.

In this study, the four groups of rat intestinal epithelial tissues were examined by immunohistochemical to detect occludin expression levels. The results showed that neither the EE nor probiotics had any significant effect on the secretion of occludin, nor was there any difference in the expression of occludin between the four groups. Some studies have shown that long duration and high intensity exercise will destroy the TJ of the intestinal tract. Active occludin is an important part of intestinal TJs ${ }^{[43]}$. All three parts of the rich environment can produce physical movement ${ }^{[20]}$. Therefore, there is no significant effect on occludin in the development of colon cancer. The regulatory effect of probiotics on the intestinal mechanical barrier of CRC is mainly produced by enhancing the gene expression of TJ proteins such as occludin ${ }^{[44]}$. However, some studies have shown that two weeks of intervention time is too short and has no obvious protective effect on intestinal mucosal barrier ${ }^{[45]}$. The results of our study are similar to this conclusion. Therefore, a longer intervention time should be used in further studies.

The effects of EE on intestinal mucosal morphology, whether intestinal epithelial villus length, intestinal mucosa thickness, or muscle thickness, were all better than those of the probiotics group, suggesting that a probiotics intervention for two weeks in advanced CRC has a limited effect on the intestinal mucosal mechanical barrier. Treatment with probiotics was not able to resist the damage caused by tumor's growth and consumption to the intestinal structure of the body and did not have a synergistic effect with $E E$. In previous studies on the effect of probiotics on colorectal cancer, most of the subjects focused on patients with colorectal cancer after operation ${ }^{[46]}$. In the case of tumor resection, probiotics have a protective effect on intestinal mucosal mechanical barrier. However, this study did not remove the tumors, and probiotics did not play a significant role. Therefore, we speculate that the reason may be that the regulation of probiotics can not completely resist the damage of intestinal structure caused by the growth and consumption of tumors, and can not form a more effective synergistic effect with the rich environment. Social support, cognitive stimulation and physical movement in rich environments may lead to mechanical changes of intestinal mucosa by adjusting brain-gut axis or micro-environment, which may play a more effective role in maintaining or promoting the integrity of intestinal mucosal mechanical barrier ${ }^{[47]}$. 
Therefore, in the regulation of intestinal mucosal mechanical barrier, the role of enriched environment is better than that of probiotics and the interaction between them. However, the effect of occludin on intestinal pathomorphology needs further study.

\section{Intestinal Mucosal Immune Barrier}

Cytokines have a central role in systemic changes in cancer patients ${ }^{[13]}$. Moreover, they are the major regulators of mucosal immunity and play an important part in the intestinal immune defense. Cancer patients generally have changes in cytokine levels, which seriously affect the metabolism and immunity of the body.

There are two main types of cytokine: (1) factors that promote the inflammatory reaction, such as TNF-a, IL-1, and IL-6; and (2) suppression of inflammatory response factors, such as IL-4 and IL-10 [48]. Our results showed that the EE can regulate cytokines in both serum and intestinal mucosal; specifically, it can adjust IL-10, IL-6, and TNF-a, with beneficial effects on the body. Probiotics also play a part in regulating serum cytokines, but their beneficial effect on the body is weaker than that of the EE. The combination of probiotics and EE can produce an interaction, sometimes with a beneficial effect on the body; however, sometimes the combined effect is weaker than that of a single factor or may even have a negative effect on the body. Overall, the results show that EE can regulate the immune function of the intestinal mucosal immune barrier, with a more marked regulatory effect in the serum and intestinal mucosa IL-10. This is similar to the results of other studies, which showed that ${ }^{[49]} \mathrm{EE}$ as a benign pressure can regulate the level of adiponectin by regulating the hypothalamus sympathetic nerve cell axis, thus affecting the secretion of cytokines. The effect of the single use of probiotics in immune factor regulation is weaker than that of environmental enrichment. However, the interaction of probiotics and EE on cytokines needs further study.

SIgA is the most secreted immunoglobulin in the body and also an important part of the intestinal immune barrier. SIgA is resistant to proteolysis in the gut and does not activate the alexine and inflammatory reaction. SIgA is an ideal protective agent for intestinal mucosa and has an important role in determining the composition of the intestinal mucosal immune barrier. Therefore, SIgA can be used to evaluate the function of the intestinal mucosal immune barrier $[40,50,51]$. The results of this study showed that the EE and probiotics had no obvious effect on SIgA secretion. Upon further comparison, there were no significant differences between the different groups. Therefore, we cannot conclude that EE and probiotics play a part in the secretion of intestinal immunoglobulins. This may be because the intervention time was too short. A longer intervention time should be used in future studies to investigate the effects of EE and probiotics on SIgA.

The EE can directly or indirectly regulate the hypothalamic-pituitary-adrenal and hypotha-lamicsympathoneural-adipocyte(HSA) axes, by utilizing the humoral system, neuroendocrine system, and immune system; regulating the gene expression of the hypothalamus; and promoting the proliferation of 
splenic lymphocytes and the mitosis of T cells. In this way, it can regulate serum and intestinal mucosal cytokine levels to protect the intestinal mucosal immune barrier ${ }^{[5]}$. Probiotics can directly affect the intestinal tract, strengthening the activity of macrophages and other non-specific defense functions, thus protecting the intestinal mucosal immune barrier. However, their effect was weaker than that of $E E$ on the body fluid-endocrine system. However, the production of SIgA is the result of a synergistic effect of B cells, $T$ cells, and cytokines of the local microenvironment ${ }^{[52]}$. The regulation mechanism of external factors needs further study.

\section{Intestinal Mucosal Biological Barrier}

Generally, BT refers to the translocation of intestinal bacteria from the intestinal lumen to the mesentery or other organs. Under normal conditions, intestinal BT does not occur easily, owing to tight intestinal junctions. However, BT increases during bacterial pathogenesis in the intestinal tract or during periods of stress, when the mucosal epithelium is damaged. Therefore, BT can be used to evaluate the permeability of the intestinal mucosal barrier ${ }^{[53]}$. In this study, we found that there was no combined effect of $E E$ and probiotics on BT; however, analysis of the individual factors showed that environmental enrichment and probiotics could both affect BT. Upon further analysis, the BT rate in the EE group was found to be lower than that of group $\mathrm{P}$, indicating that the protection by probiotics of the intestinal mucosa biological barrier in $\mathrm{CRC}$ is limited compared with the protection by EE. The effect of EE on the intestinal mucosa biological barrier is thus more beneficial. Probiotics can decrease intestinal dysbacteriosis and the BT rate, and enhance the effect of resistance to pathogens, thereby protecting the intestinal barrier, inhibiting tumor growth and reducing intestinal complications. However, in this study, we found that the role of probiotics was not as important as that of the EE in CRC. A possible reason is that stress can reduce intestinal bacteria, such as lactic acid bacteria, while eustress can increase intestinal bacteria, prevent

dysbacteriosis, and promote TJs of the intestinal mucosa, thereby reducing the incidence of $B T^{[54]}$. EE, as a kind of eustress stimulation ${ }^{[49]}$, also helps to maintain the biological barrier function of the intestinal mucosa.

\section{Brain-gut Peptides}

$\mathrm{CRF}$ is the main mediator for the central nervous system to participate in stress response. Under stress conditions such as diseases, CRF can be overexpressed to regulate gastrointestinal motility, secretion and sensation through the HPA axis[55]. Ghrelin is an endogenous brain-gut peptide composed of 28 amino acids discovered by Japanese scientist Kojima in 1999[56].The binding of Ghrelin with its receptor can produce a wide range of biological effects, such as stimulating the secretion of growth hormone, regulating food intake and energy metabolism, regulating immune function, protecting gastrointestinal mucosa, regulating gastrointestinal motility, promoting gastric acid secretion, controlling the proliferation of gastrointestinal cancer cells and improving gastrointestinal dysfunction[57]. Therefore, both 
corticotropin-releasing factor and Ghrelin can be used as important indicators for evaluating brain-gut axis function.

In the regulation of $\mathrm{CRF}, \mathrm{EE}$ and probiotics could cross-react. Both $\mathrm{EE}$ and probiotics were beneficial to the secretion of CRF, but the effect of EE was greater than that probiotics, and the interaction of EE and probiotics was similar to the effect of probiotics alone.

Regarding the secretion of ghrelin, the intervention condition only influenced the secretion of hypothalamic ghrelin and had no effect on the secretion of intestinal mucosa ghrelin. There was no effect of the interaction on hypothalamic ghrelin secretion, but EE and probiotic separately can both effect the secretion of hypothalamic ghrelin. The effect of EE was greater than that of probiotics. Through two-two comparision, there was no significant effect on hypothalamic ghrelin secretion when using probiotics alone. When probiotics were combined with $\mathrm{EE}$, the effect became more marked.

Therefore, in the study of brain-gut peptides, the role of EE is greater than that of probiotics. Studies have shown that both inside and outside body pressure and cognitive disorders affect the brain-gut axis, damaging the intestinal mucosal barrier with detrimental effects on its function. The cognitive training, social support, and physical exercise involved in the EE help to relieve body pressure and cognitive impairment ${ }^{[58,59]}$, thus protecting the intestinal mucosal barrier. In addition, some studies have shown that probiotics also modulate brain activity $[22,60]$, although our results suggest that this effect is less beneficial compared with environmental enrichment.

In summary, in the study of the intestinal mucosal barrier and brain-gut peptides, the effect of EE was greater than that of probiotics. However, the combined effect was not better than that of EE alone. In future studies, we intend to investigate the role of the environment and probiotics in determining SIgA levels, body weight, and the intestinal mucosal mechanical barrier by extending the intervention time.

\section{Body Weight}

There were no significant differences in body weight between different groups or within groups. The reason for this may be the short intervention time. In addition, we did not measure tumor weight. This was because the tumors in the rat were mostly multiple after the rats were dissected, which made it difficult to measure the weight of tumors. Therefore, to understand the effects of this intervention on body weight, further studies are needed with longer duration and deeper observation.

\section{Conclusions}

In the study of intestinal mucosal barrier and brain-gut peptide, the role of enriched environment is better than probiotics, but the interaction between them is not as good as that of enriched environment alone. Therefore, environmental nursing is very important for the rehabilitation of colorectal cancer, and the interaction between environmental nursing and probiotics is not significant, nor is it obvious for the body. 
In the future, we can further study the effects of enriched environment and probiotics on the body by prolonging intervention time and combining rehabilitation nursing, and further study the mechanism and approach of enriched environment on intestinal mucosal barrier.

\section{Declarations}

\section{Ethical Approval and Consent to participate}

Specific Pathogen Free (SPF) male Sprague-Dawley (SD) rats were purchased from the animal experiment center of Fujian Medical University. Rats were purchased with the license number SCXK (Fujian) 2016-0002. After the purchase, rats were reared at the Animal Experiment Center of Fujian Medical University, using the license number SYXK (Fujian) 2016-0006. All procedures performed in studies involving animals were in accordance with the ethical standards of the institution or practice at which the studies were conducted (Fujian Medical University, 2016-06).

\section{Consent for publication}

Not applicable.

\section{Availability of supporting data}

Others can be able to replicate and build upon the authors' published claims. Authors can make materials, data, code, and associated protocols promptly available to readers without undue qualifications. Any restrictions on the availability of materials or information must be disclosed to the editors at the time of submission. Any restrictions must also be disclosed in the submitted manuscript.

\section{Authors' contributions and Conflict of Interest}

All authors participated in the design, interpretation of the studies and analysis of the data and review of the manuscript; Wu Xian-Yi conducted the experiments, Liu Dun supplied critical reagents and animals, Liu Dun and Huang Si-ting wrote the manuscript. The authors declare that they have no conflict of interest.

\section{Funding}

This study was funded in full by Fujian Province Youth Teacher Education Research Project of China (grant no.JA17022) and Fujian Provincial Joint innovation project of science and Technology Department (grant no. 2018Y9101).

\section{Acknowledgements}

We thank Mr Xiang-xin Wu for technical assistant. We also grateful to prof Cai-hua Huang for her advices on EE housing setting up. 


\section{References}

1. Jadavji NM, Kolb B, Metz GA. Enriched environment improves motor function in intact and unilateral dopamine-depleted rats. Neuroscience. 2006;140:1127-38.

2. Wu Y, Gan Y, Yuan H, Wang Q, Fao Y, Li G, et al. Enriched environment housing enhances the sensitivity of mouse pancreatic cancer to chemotherapeutic agents. Biochem Biophys Res Commun. 2016;473(2):593-9.

3. Slater AM, Cao L. A protocol for housing mice in an enriched environment. J Vis Exp. 2015;100:e52874.

4. Nachat-Kappes R, Pinel A, Combe K, Lamas B, Farges M-C, Rossary A, et al. Effects of enriched environment on COX-2, leptin and eicosanoids in a mouse model of breast cancer. PLoS One. 2012;7(12):e51525.

5. Cao L, Liu X, Lin E-JD, Wang C, Choi EY, Riban V, et al. Environmental and genetic activation of a brain-adipocyte BDNF/leptin axis causes cancer remission and inhibition. Cell. 2010;142:52-64.

6. Chen $\mathrm{H}$, Yang $\mathrm{Y}$. The effect of oral probiotics on the inflammatory reaction and intestinal barrier function after colorectal cancer surgery (in Chinese). Zhong Guo Zhong Xi Yi Jie He Xiao Hua Za Zhi. 2014; 22(1): 38-40.

7. Kotzampassi K, Stavrou G, Damoraki G, Georgitsi M, Basdanis G, Tsaousi G, et al. A four-probiotics regimen reduces postoperative complications after colorectal surgery: a randomized, double-blind, placebo-controlled study. World J Surg. 2015. (doi: 10.1007/s00268-015-3071-z).

8. Liu YS, Shao B, Zhang B, Wang LB, Cai P, Liu FL. Study on the changes of intestinal flora and effect of micro-ecological agents in colorectal cancer patients (in Chinese). Chinese Rural Health Service Administration. 2013;33(5):584-6.

9. Ding HD, Su Q, Yin HZ. Use of immune microecological nutrition as an alternative to traditional bowel preparation in minimally invasive treatment of colorectal cancer (in Chinese). J Chin Digest Tract. 2013;21(10):940-4.

10. Liu ZC, Wang LP, Li HP. Application of immune micro-ecological nutrition instead of traditional bowel preparation in colorectal cancer (in Chinese). Chinese Modern Doctor. 2013;51(31):18-22.

11. Yang L, Yang JY. Clinical study of colorectal cancer perioperative application of intestinal probiotics affecting postoperative inflammation and infectious complications (in Chinese). Chinese Journal of Gastroenterol Hepatol. 2014;23(11):1302-4.

12. Yu JT. Effect on immune function of preoperative enteral immune nutrition joint with probiotics in patients with colorectal tumor. Masters thesis (in Chinese), Guangxi Medical University, China. 2008.

13. Jia Y. Study on the injury and protection of intestinal mucosa cancerous malnutrition. Doctoral thesis (in Chinese), Second Military Medical University, Shanghai, China. 2005.

14. Olejnik A, Tomczyk J, Kowalska K, Grajek W. The role of natural dietary compounds in colorectal cancer chemoprevention (in Polish). Postepy Hig Med Dosw. 2010;64:175-87. 
15. She J, Li T. Intestinal mucosal immune function changes after colorectal cancer surgery (in Chinese). Zhong Guo Xian Dai Yi Yao Za Zhi. 2008;10(6):20-4.

16. Tlaskalova-Hogenova H, Stepankova R, Kozakova H, Hudcovic T, Vannucci L, Tuckova L, et al. The role of gut microbiota (commensal bacteria) and the mucosal barrier in the pa hogenesis of inflammatory and autoimmune diseases and cancer: contribution of germ-free and gnotobiotic animal models of human diseases. Cell Mol Immunol. 2011;8:110-20.

17. Vannucci L, Stepankova R, Kozakova H, Fiserova A, Rossmann P, Tlaskalova-Hogenova H. Colorectal carcinogenesis in germ-free and conventionally reared rats: different intestinal environments affect the systemic immunity. Int J Oncol. 2008;32:609-17.

18. Wang L, Pan L, Shi L, Sun Y, Zhang Y, Zhou D. Effect of complete peptide on LPS activation of peritoneal macrophages in nude mice by Bacillus (in Chinese). Chin J Microbiol Immunol. 2000;20(1):4-6.

19. Ye Y. National Clinical Laboratory operation regulations (version 2) (in Chinese). NanJing: Southeast University Press; 1997.

20. Xie H, Wua Y, Jia J, Liu G, Zhang Q, Yua K, et al. Enrichment-induced exercise to quantify the effect of different housing conditions: A tool to standardize enriched environment protocols. Behav Brain Res. 2013;249:81-9.

21. Su LK, Kinzler KW, Vogelsterin B, Preisinger AG, Moser AR, Luongo C, et al. Multiple intestinal neoplasia caused by a mutation in the murine homolog of the APC gene. Science. 1992;256:668-70.

22. Tillisch K, Labus J, Kilpatrick L, Jiang Z, Stains J, Ebrat B, et al. Consumption of fermented milk product with probiotic modulates brain activity. Gastroenterology. 2013;144:1394-401.

23. Davari S, Talaei SA, Alaei H, Salami M. Probiotics treatment improves diabetes-induced impairment of synaptic activity and cognitive function: behavioral and electrophysiological proofs for microbiome-gut-brain axis. Neuroscience. 2013;240:287-96.

24. Chuan Q. Medical laboratory animal science [M]. Beijing: People's Health Press; 2008.

25. Pugh S, Thomas GA. Patients with adenomatous polyps and carcinomas have increased colonic mucosal prostaglandin E2. Gut. 1994;35:675-8.

26. Furuta Y, Hall ER, Sanduja S, Barkley T Jr, Milas L. Prostaglandinproduction by murine tumors as a predictor for theraoeutic response to indomethacin. Cancer Res. 1988;48:3002-7.

27. Martinez ME, Heddens D, Earnest DL, Bogert CL, Roe D, Einspahr J, et al. Physical Activity, Body Mass Index, and Prostaglandin E2 levels in Rectal Mucisa. J Natl Cancer Inst. 1999;91(11):950-3.

28. Nithianantharajah J, Hannan AJ. Enriched environments, experience- dependent plasticity and disorders of the nervous system. Nat Rev Neurosci. 2006;7:697-709.

29. He ZM, Li GP, Zhu DS, Lu SM. Guidelines for the management and use of experimental animals (In Chinese). Beijing: Science Press; 2016.

30. Dellen A, Blakemore C, Deacon R, York D, Hannan AJ. Delaying the onset of Huntington's in mice. Nature. 2000;404:721-2. 
31. Spires TL, Grote HE, Varshney NK, Cordery PM, van Dellen A, Blakemore C, et al. Environmental enrichment rescues protein deficits in a mouse model of Huntington's disease, indicating a possible disease mechanism. J Neurosci. 2004;24:2270-6.

32. Wu YT. Research on protective effect of probiotics to 5-Fu induced rats' intestinal mucosa injury. Masters thesis (in Chinese), Dalian Medical University, China. 2014.

33. Zheng XZ. Observation on the curative effect of probiotics on colitis in rats and Study on the changes of intestinal flora. Master thesis (in Chinese), Kunming Medical University, 2015.

34. Sheng WQ, Ming ZZ, Yi G. Practical medical media manuals [M]. Beijing: People's Military Medical Press; 1999.

35. Chiva M, Soriano G, Rochat I, Peralta C, Rochat F, Llovet T, et al. Effects of lactobacillus johnsonii La1 and antioxidants in intestinal flora and bacterial translocation in rats with experimental cirrhosis. $\mathrm{J}$ Hepatol. 2002;37:456-62.

36. Shimizu M, Son DO. Food-derived peptides and intestinal functions. Curr Pharm Des. 2007;13(19):885-95.

37. Guarner F, Malagelada JR. Gut flora in health and disease. Lancet. 2003;361:512-9.

38. Lim CC, Ferguson LR, Tannock GW. Dietary fibres as "prebiotics": Implications for colorectal cancer. Mol Nutr Food Res. 2005;49:609-19.

39. van Praag H, Kempermann G, Gage FH. Neural consequences of environmental enrichment. Nat Rev Neurosci. 2000;1:191-8.

40. Yang Z. Effect of microecological agent on inflammatory response and intestinal barrier function in patients with colorectal carcinoma. Masters thesis (in Chinese), Shanghai Jiao Tong University, China. 2007.

41. Liu Z, Qin H, Yang Z, Xia Y, Liu W, Yang J, et al. Randomised clinical trial: the effects of perioperative probiotic treatment on barrier function and post-operative infectious complications in colorectal cancer surgery - a double-blind study. Aliment Pharmacol Ther. 2011;33:50-63.

42. Zhang MM. Ecoimmunonutrition Management after surgical operation: basic and clinical research. Doctoral thesis (in Chinese), Sichuan University, China. 2007.

43. Zuhl M, Schneider S, Lanphere K, Conn C, Dokladny K, Moseley P. Exercise regulation of intestinal tight junction proteins. Sports Med. 2014;48:980-6.

44. Chong ES. A potential role of probiotics in colorectal cancer prevention: review of possible mechanisms of action. World J Microbiol Biotechnol. 2014;30:351-74.

45. McNaught CE, Woodcock NP, MacFie J, Mitchell CJ. A prospective randomised study of the probiotic Lactobacillus plantarum $299 \mathrm{~V}$ on indices of gut barrier function in elective surgical patients. Gut. 2002;51:827-31.

46. Dun Liu X-Y, Jiang L-S, Zhou MD, PhD J-H, Song, Zhang X. MD. Effects of Probiotics on Intestinal Mucosa Barrier in Patients with Colorectal Cancer after Operation Meta-Analysis of Randomized Controlled Trials. Medicine(Baltimore). 2016V95N15:e3342. 
47. Bonaz BL, Bernstein CN. Brain-gut interactions in inflammatory bowel disease. Gastroenterology. 2013;144:36-49.

48. Jones SA, Butler RN, Sanderson IR, Wilson JW. The effect of specific caspase inhibitors on TNF-a and butyrate-induced apoptosis of intestinal epithelial cells. Exp Cell Res. 2004;292:29-39.

49. Cao L, During MJ. What is the brain-cancer connection? Annu Rev Neurosci. 2012;35:331-45.

50. Zhang JW, Du P, Gao J, Yang BR, Fang WJ, Ying CM. Preoperative probiotics decrease postoperative infectious complications of colorectal cancer. Am J Med Sci. 2012;343:199-205.

51. Zhang JW, Du P, Chen DW, Cui L, Ying CM. Effect of viable Bifidobacterium supplement on the immune status and inflammatory response in patients undergoing resection for colorectal cancer (in Chinese). Zhonghua Wei Chang Wai Ke Za Zhi. 2010;13(1):40-3.

52. Zan P, Honghu C. Research progress on the production mechanism of secretory immunoglobulin A in intestinal mucosa (in Chinese). J Xinxiang Med Coll. 2011;28(6):773-5.

53. Zhang X. A correlation study between enteral nutrition of adding different nutrients to improve mucosa barrier function and dopamine receptor expression of brain injury; rat. Doctoral thesis (in Chinese), Fujian Medical University, China. 2012.

54. Galley JD, Nelson MC, Yu Z, Dowd SE, Walter J, Kumar PS, et al. Exposure to a social stressor disrupts the community structure of the colonic mucosa-associated microbiota. BMC Microbiol. 2014;14:189.

55. Zhu WL. Effect mechanism of electroacupuncture at different meridians on brain-gut axis-related neuropeptide in rats with irritable bowel syndrome. Doctoral thesis (in Chinese), Beijing University of Traditional Chinese Medicine, China. 2012.

56. Ghrelin is a

Kojima M, Hosoda H, Date Y, Nakazato M, Matsuo H, Kangawa K. Ghrelin is a growth-hormonereleasing acylated peptide from stomach. Nature. 1999;402:656-60.

57. Liu WA. To explore the mechanism of herbal cake-separated moxibustion on gastric motility disturbance and sensory abnormality in FGIDs model rats with liver depression and spleen deficiency. Doctoral thesis (in Chinese), Hunan University of Traditional Chinese Medicine, China. 2013.

58. Pigrau M, Rodino-Janeiro BK, Casado-Bedmar M, Lobo B, Vicario M, Santos J, et al. The joint power of sex and stress to modulate brain-gut-microbiota axis and intestinal barrier homeostasis: implications for irritable bowel syndrome. Neurogastroenterol Motil. 2015. (doi: 10.1111/nmo.12717).

59. Caracciolo B, Xu W, Collins S, Fratiglioni L. Cognitive decline, dietary factors and gut-brain interactions. Mech Ageing Dev. 2014;136-137:59-69.

60. Quigley EM, Shanahan F. The future of probiotics for disorders of the brain-gut axis. Adv Exp Med Biol. 2014. (doi: 10.1007/978-1-4939-0897-4-19).

\section{Tables}


Due to technical limitations, Tables 1-4 are provided in the Supplementary Files section.

\section{Figures}
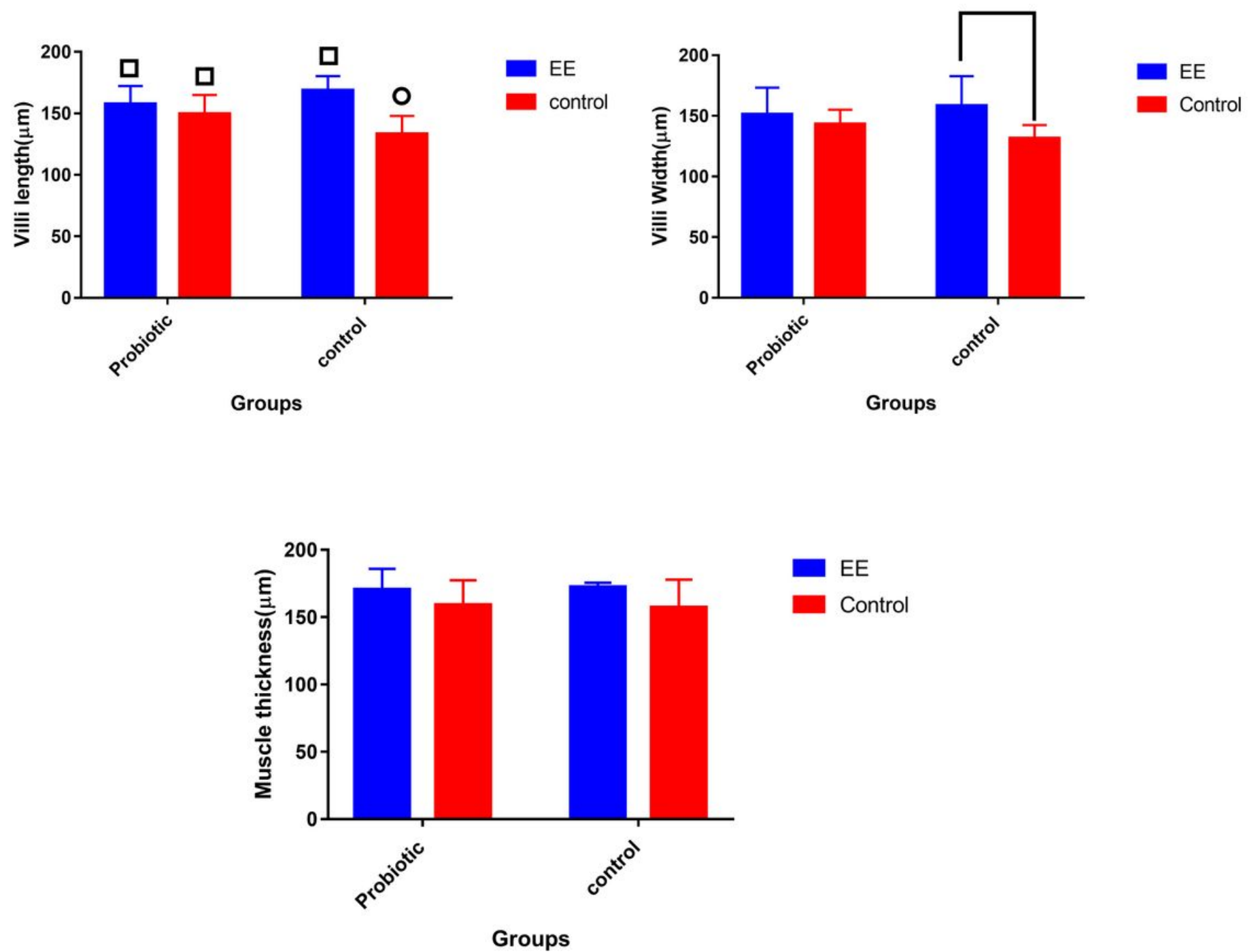

Figure 1

Microscopic morphology of intestinal mucosa in eight groups of rats $\square p>0.05$ compared between each other with the same symbol Othe lowest level group compared with all other groups $\Pi p<0.05$ compared between each other with the same symbol; 

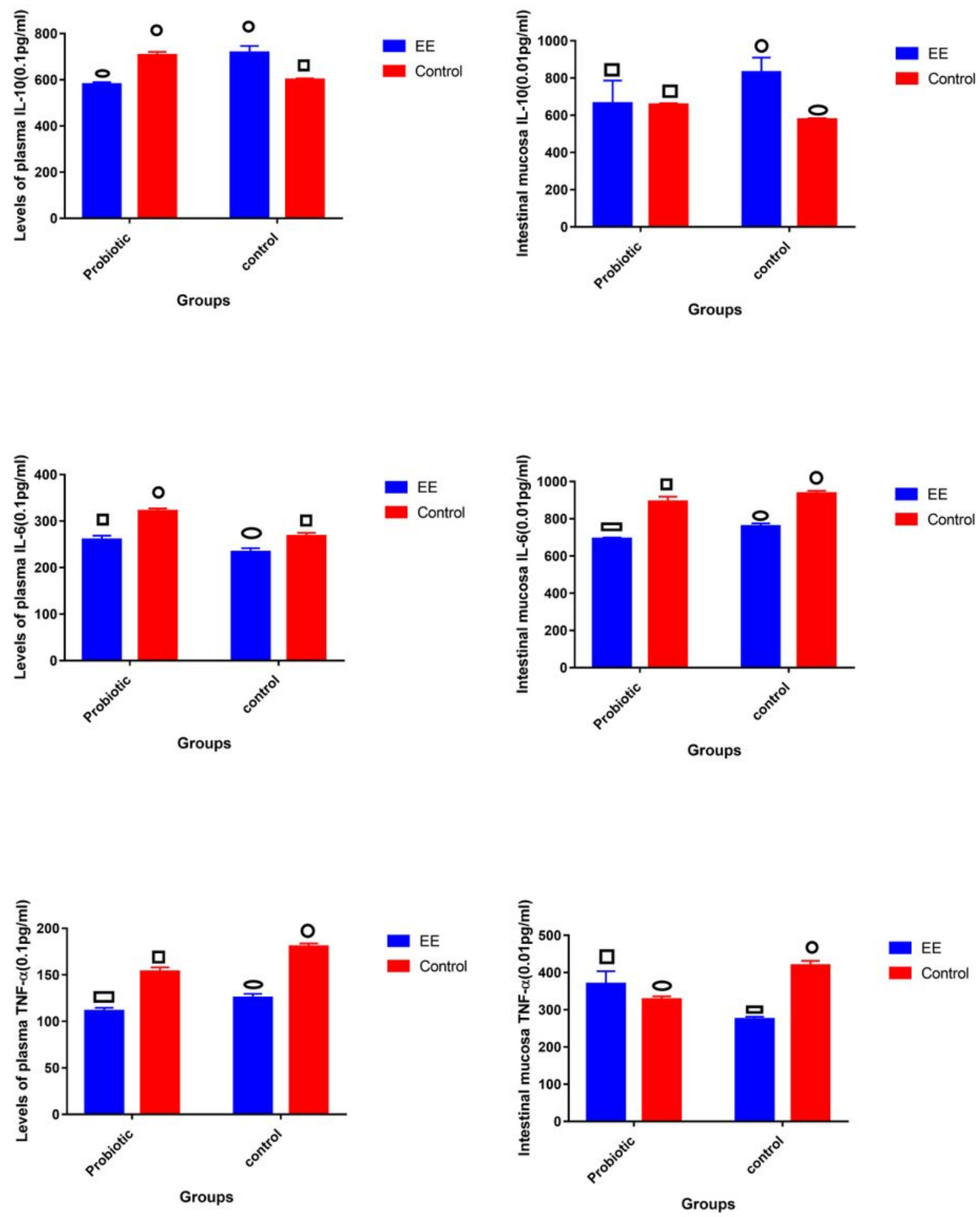

Figure 2

Plasma and intestinal mucosal TNF-a, IL-6, and IL-10 levels in four groups of rats with colorectal cancer. Different shapes represent $P>0.05$ 


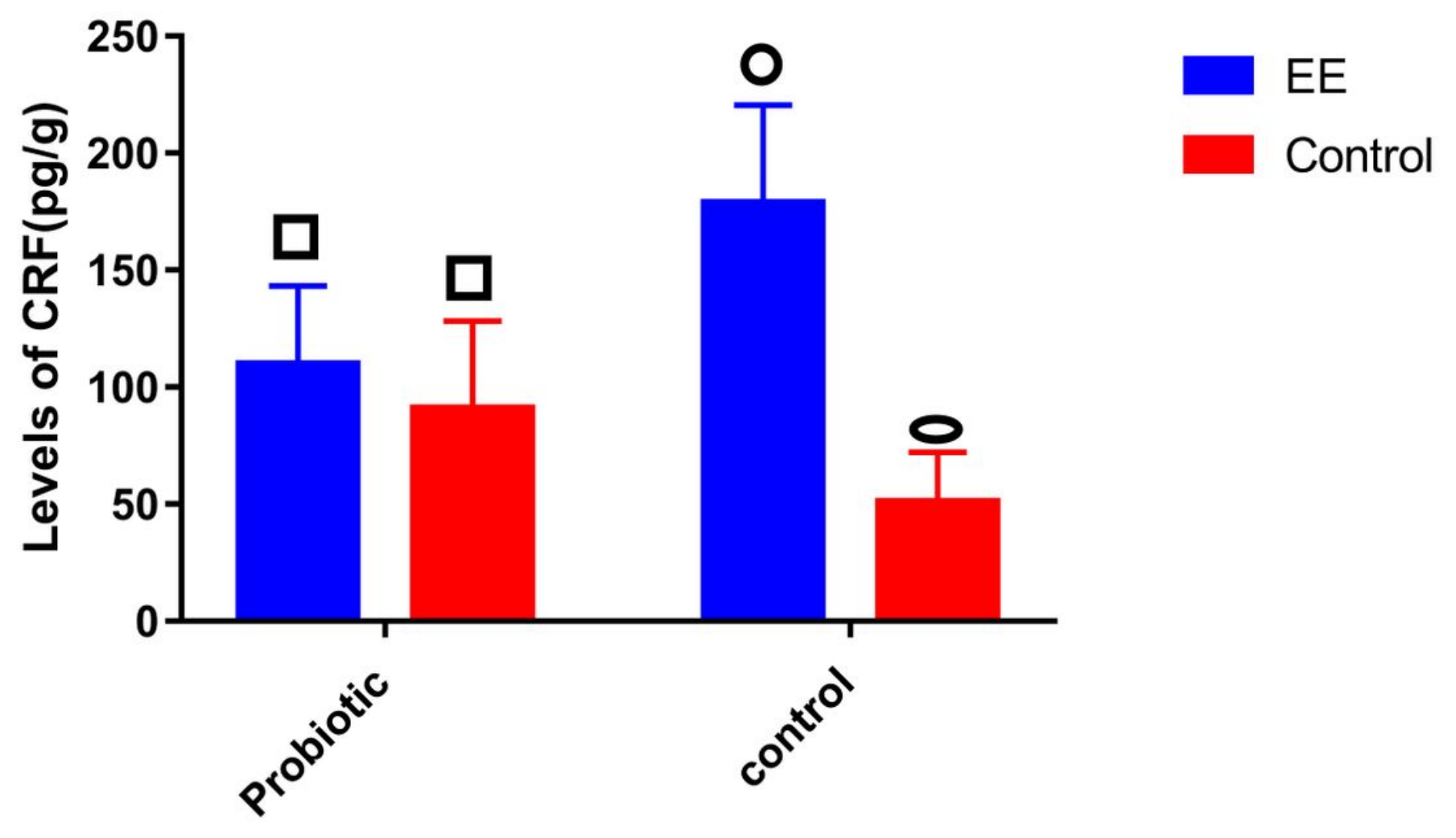

Groups

Figure 3

CRF levels in the hypothalamus of rats with colorectal cancer. Different shapes represent $P>0.05$ 

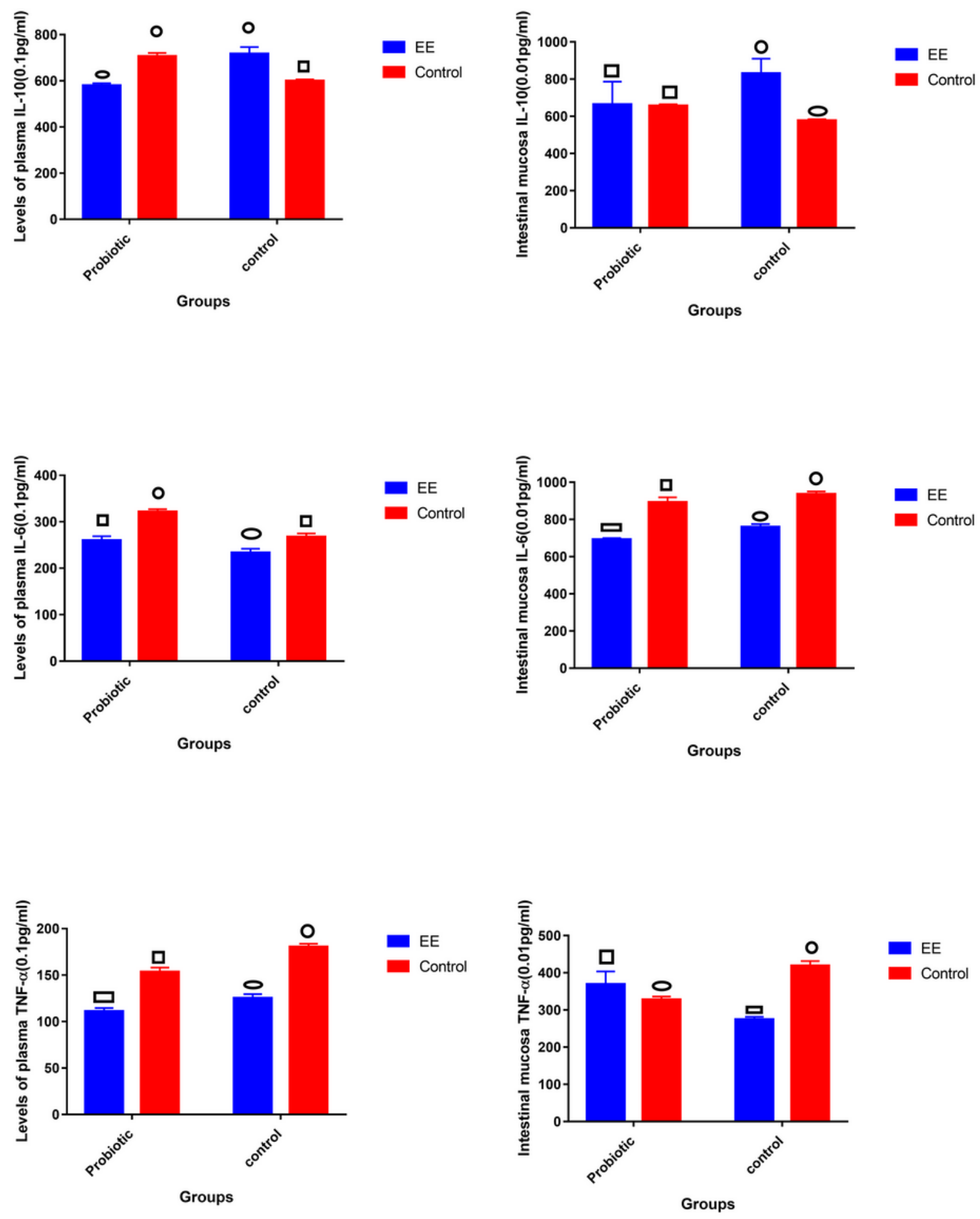

Figure 4

Ghrelin secretion in intestinal mucosa and hypothalamus. Different shapes represent $P>0.05$

\section{Supplementary Files}

This is a list of supplementary files associated with this preprint. Click to download. 
- table2.doc

- table3.doc

- table4.doc

- table1.doc 